\title{
PSEUDO-RIEMANNIAN MANIFOLDS WITH COMMUTING JACOBI OPERATORS
}

\author{
M. BROZOS-VÁZQUEZ AND P. GILKEY
}

\begin{abstract}
We study the geometry of pseudo-Riemannian manifolds which are Jacobi-Tsankov, i.e. $\mathcal{J}(x) \mathcal{J}(y)=\mathcal{J}(y) \mathcal{J}(x)$ for all $x, y$. We also study manifolds which are 2-step Jacobi nilpotent, i.e. $\mathcal{J}(x) \mathcal{J}(y)=0$ for all $x, y$.
\end{abstract}

\section{INTRODUCTION}

Let $\mathcal{M}:=(M, g)$ be a pseudo-Riemannian manifold of signature $(p, q)$ and dimension $m=p+q \geq 3 ; \mathcal{M}$ is said to be Riemannian if $p=0$ and Lorentzian if $p=1$. Although the Riemannian and Lorentzian settings are perhaps the most frequently studied, pseudo-Riemannian manifolds with other signatures are important in many physical applications; see, for example, the discussion of Kaluza-Klein gravity in Overduin and Wesson [15] or the brane world cosmology of Shtanov and Sahni [16. Thus the higher signature setting is important not only mathematically, but also in physical applications.

Let $\mathcal{R}$ be the curvature operator and $\mathcal{J}$ the Jacobi operator which are defined by the Levi-Civita connection on $\mathcal{M}$ :

$$
\begin{aligned}
& \mathcal{R}(x, y):=\nabla_{x} \nabla_{y}-\nabla_{y} \nabla_{x}-\nabla_{[x, y]}, \\
& \mathcal{J}(x): y \rightarrow \mathcal{R}(y, x) x .
\end{aligned}
$$

The relationship between the spectral geometry of $\mathcal{J}$ and the underlying geometry of the manifold has been studied extensively in recent years. Suppose that $\mathcal{M}$ is Riemannian. If $\mathcal{M}$ is a 2-point homogeneous space, then the group of isometries acts transitively on the unit sphere bundle $S(\mathcal{M})$ and hence the eigenvalues of $\mathcal{J}$ are constant on $S(\mathcal{M})$. Osserman [14] wondered if the converse is true, at least locally. He conjectured that if $\mathcal{M}$ is a Riemannian manifold such that the eigenvalues of $\mathcal{J}$ are constant on $S(\mathcal{M})$, then either $\mathcal{M}$ is flat or $\mathcal{M}$ is locally isometric to a rank 1 -symmetric space. This conjecture has been established in dimensions $m \neq 16$ by the work of Chi $[6$ and Nikolayevsky [12, 13; the case $m=16$ is still open.

Let $S^{ \pm}(\mathcal{M})$ be the pseudo-sphere bundles of unit spacelike $(+)$ or unit timelike $(-)$ vectors. One says that a pseudo-Riemannian manifold $\mathcal{M}$ is spacelike Osserman (resp. timelike Osserman) if the eigenvalues of the Jacobi operator $\mathcal{J}$ are constant on $S^{+}(\mathcal{M})$ (resp. on $S^{-}(\mathcal{M})$ ). Work of García-Río et. al. 8 shows these are equivalent concepts so one simply speaks of an Osserman manifold. It is known [2] 8] that any Lorentzian Osserman manifold has constant sectional curvature; thus the geometry is very rigid in this setting. However if $p \geq 2$ and $q \geq 2$, there are Osserman pseudo-Riemannian manifolds which are not locally homogeneous; see, for example, [3, 7.

One can weaken this condition slightly. Let $p \geq 1$ and $q \geq 1$. One says that $\mathcal{M}$ is pointwise Osserman if the spectrum of $\mathcal{J}$ is constant on $S_{P}^{+}(\mathcal{M})$, or equivalently on $S_{P}^{-}(\mathcal{M})$, for every $P \in M$. Blažić [1] has shown that if the spectrum of $\mathcal{J}$ is bounded on either $S_{P}^{+}(\mathcal{M})$ or, equivalently, $S_{P}^{-}(\mathcal{M})$, for every $P \in M$, then necessarily $\mathcal{M}$ is pointwise Osserman.

Key words and phrases. Jacobi operator, Jacobi-Tsankov manifold. 2000 Mathematics Subject Classification. 53C20. 
In this paper, instead of focusing on the spectrum, we will relate commutativity properties of $\mathcal{J}$ to the underlying geometry.

Definition 1.1. One says that a pseudo-Riemannian manifold $\mathcal{M}$ is:

(1) 2-step Jacobi nilpotent if $\mathcal{J}(x) \mathcal{J}(y)=0$ for all tangent vectors $x, y$.

(2) Jacobi-Tsankov if $\mathcal{J}(x) \mathcal{J}(y)=\mathcal{J}(y) \mathcal{J}(x)$ for all tangent vectors $x, y$.

(3) Orthogonally Jacobi-Tsankov if $\mathcal{J}(x) \mathcal{J}(y)=\mathcal{J}(y) \mathcal{J}(x)$ for all $x \perp y$.

Clearly $(1) \Rightarrow(2) \Rightarrow(3)$. The following seminal result was established by Tsankov 17:

Theorem 1.2. Let $\left\{\lambda_{i}\right\}$ be the eigenvalues of the shape operator of a hypersurface $M$ in $R^{m+1}$. Then $M$ is orthogonally Jacobi-Tsankov if and only if either $\lambda_{1}=$ $\ldots=\lambda_{m}$ or $\lambda_{1}=\ldots=\lambda_{m-1}=0, \lambda_{m} \neq 0$.

Theorem 1.2 has been extended from hypersurfaces to the more general setting in [5]:

Theorem 1.3. Let $\mathcal{M}$ be an orthogonally Jacobi-Tsankov Riemannian manifold. Then $\mathcal{M}$ has constant sectional curvature.

In passing to more general signatures, we shall impose a stronger condition and study Jacobi-Tsankov manifolds. It is convenient to work in the algebraic context. Let $V$ be a finite dimensional real vector space. Let $\mathfrak{A}(V) \subset \otimes^{4} V^{*}$ be the space of algebraic curvature tensors; these are the 4 -tensors with the same symmetries as the Riemann curvature tensor. Thus $A \in \mathfrak{A}(V)$ if and only if we have the following symmetries for all $x, y, z, w \in V$ :

$$
\begin{aligned}
& A(x, y, z, w)=-A(y, x, z, w)=A(z, w, x, y), \\
& A(x, y, z, w)+A(y, z, x, w)+A(z, x, y, w)=0 .
\end{aligned}
$$

Let $\mathfrak{M}:=(V,\langle\cdot, \cdot\rangle, A)$ where $A \in \mathfrak{A}(V)$ and where $\langle\cdot, \cdot\rangle$ is a non-degenerate symmetric bilinear form of signature $(p, q)$ on $V$ which is used to raise and lower indices. The corresponding algebraic curvature operator $\mathcal{A} \in V^{*} \otimes V^{*} \otimes \operatorname{End}(V)$ is characterized by

$$
\langle\mathcal{A}(x, y) z, w\rangle=A(x, y, z, w)
$$

and the Jacobi operator $\mathcal{J}=\mathcal{J}_{A}$ is given by $\mathcal{J}(x): y \rightarrow \mathcal{A}(y, x) x$. The notions of Definition 1.1 then extend to the algebraic setting. In Section 2] we will show that:

Theorem 1.4. Let $\mathfrak{M}$ be Jacobi-Tsankov. Then:

(1) $\mathcal{J}(x)^{2}=0$ for all $x \in V$.

(2) $\mathfrak{M}$ is Osserman.

(3) If $V$ is Riemannian or Lorentzian, then $A=0$.

We can draw the following geometrical consequence from Theorem 1.4

Corollary 1.5. Let $\mathcal{M}$ be a Jacobi-Tsankov pseudo-Riemannian manifold of signature $(p, q)$. Then $\mathcal{M}$ is nilpotent Osserman. If $p=0$ or if $p=1$, then $\mathcal{M}$ is flat.

One might conjecture that the condition $\mathcal{J}(x)^{2}=0$ for all $x \in V$ is sufficient to imply $\mathfrak{M}$ is Jacobi-Tsankov. This is in fact not the case as we will show in Lemma 2.2

It is clear that any 2-step Jacobi nilpotent algebraic curvature tensor is JacobiTsankov. In Section 3 we will show that the converse holds in low dimensions:

Theorem 1.6. Let $\mathfrak{M}$ be Jacobi-Tsankov. If $\operatorname{dim}(V) \leq 13$, then $\mathfrak{M}$ is 2 -step Jacobi nilpotent. 
The condition $\operatorname{dim}(V) \leq 13$ in Theorem 1.6 is sharp. In Lemma 3.2 we construct a Jacobi-Tsankov tensor in signature $(8,6)$, which is indecomposable and for which there exist $(x, y)$ so that $\mathcal{J}(x) \mathcal{J}(y) \neq 0$.

There are similar questions for the skew-symmetric curvature operator.

Definition 1.7. One says that $\mathfrak{M}$ is:

(1) 2-step skew-curvature nilpotent if $\mathcal{A}\left(x_{1}, x_{2}\right) \mathcal{A}\left(x_{3}, x_{4}\right)=0$ for all tangent vectors $x_{1}, x_{2}, x_{3}, x_{4}$.

(2) Skew-Tsankov if $\mathcal{A}\left(x_{1}, x_{2}\right) \mathcal{A}\left(x_{3}, x_{4}\right)=\mathcal{A}\left(x_{3}, x_{4}\right) \mathcal{A}\left(x_{1}, x_{2}\right)$ for all tangent vectors $x_{1}, x_{2}, x_{3}, x_{4}$.

Motivated by Theorem 1.6 in Section 4 we will study 2-step Jacobi nilpotent algebraic curvature tensors in relation to 2-step skew-curvature nilpotent ones. If $A_{W} \in \mathfrak{A}(W)$, we say that $\left(W, A_{W}\right)$ is indecomposable if there is no decomposition $\left(W, A_{W}\right)=\left(W_{1}, A_{1}\right) \oplus\left(W_{2}, A_{2}\right)$ where $\operatorname{dim}\left(W_{i}\right) \geq 1$. Similarly, we say that $\mathfrak{M}$ is indecomposable if there is no decomposition $\mathfrak{M}=\mathfrak{M}_{1} \oplus \mathfrak{M}_{2}$ so that $\operatorname{dim}\left(V_{i}\right) \geq 1$.

Definition 1.8. Let $A_{W} \in \mathfrak{A}(W)$. Assume that $\left(W, A_{W}\right)$ is indecomposable. Let $\left\{\bar{e}_{1}, \ldots, \bar{e}_{k}\right\}$ be a basis for an auxiliary vector space $\bar{W}$. Let

$$
\begin{aligned}
& \mathfrak{M}:=\left(W \oplus \bar{W},\langle\cdot, \cdot\rangle_{W \oplus \bar{W}}, A_{W} \oplus 0\right) \quad \text { where } \\
& \left\langle e_{i}, e_{j}\right\rangle=\left\langle\bar{e}_{i}, \bar{e}_{j}\right\rangle=0, \quad\left\langle e_{i}, \bar{e}_{j}\right\rangle=\delta_{i j} .
\end{aligned}
$$

We will establish the following classification theorem:

Theorem 1.9. The following statements are equivalent:

(1) $\mathfrak{M}$ is 2-step Jacobi nilpotent and indecomposable,

(2) $\mathfrak{M}$ is 2-step skew-curvature nilpotent and indecomposable,

(3) $\mathfrak{M}$ is isomorphic to one of the tensors described in Definition 1.8

One has the following geometrical examples which arose in the study of Osserman manifolds. We refer to [10,11 for further details.

Theorem 1.10. Let $\left(x_{1}, \ldots, x_{p}, y_{1}, \ldots, y_{p}\right)$ be coordinates on $\mathbb{R}^{2 p}$ for $p \geq 2$. Let $\psi_{i j}(x)=\psi_{j i}(x)$ be a symmetric 2 -tensor. Let

$$
g_{\psi}\left(\partial_{x_{i}}, \partial_{x_{j}}\right)=\psi_{i j}(x), \quad g_{\psi}\left(\partial_{x_{i}}, \partial_{y_{j}}\right)=\delta_{i j}, \quad g_{\psi}\left(\partial_{y_{i}}, \partial_{y_{j}}\right)=0 .
$$

Then $\mathcal{M}:=\left(\mathbb{R}^{2 p}, g_{\psi}\right)$ is a complete pseudo-Riemannian manifold of neutral signature $(p, p)$ which is 2-step Jacobi nilpotent and 2-step skew-curvature nilpotent.

\section{The Proof of Theorem 1.4}

The Jacobi operator is quadratic in $x$. We polarize to define an operator valued bilinear form by setting:

$$
\mathcal{J}(x, y):\left.z \rightarrow \frac{1}{2} \partial_{\varepsilon} \mathcal{J}(x+\varepsilon y) z\right|_{\varepsilon=0}=\frac{1}{2}\{\mathcal{A}(z, x) y+\mathcal{A}(z, y) x\} .
$$

Setting $x=y$ yields $\mathcal{J}(x, x)=\mathcal{J}(x)$. Furthermore

$$
\mathcal{J}(x, y) x=\frac{1}{2}(\mathcal{A}(x, x) y+\mathcal{A}(x, y) x)=-\frac{1}{2} \mathcal{J}(y) x
$$

Let $A$ be a Jacobi-Tsankov algebraic curvature tensor. Polarizing the identity $\mathcal{J}(x) \mathcal{J}(y)=\mathcal{J}(y) \mathcal{J}(x)$ yields:

$$
\mathcal{J}\left(x_{1}, x_{2}\right) \mathcal{J}\left(y_{1}, y_{2}\right)=\mathcal{J}\left(y_{1}, y_{2}\right) \mathcal{J}\left(x_{1}, x_{2}\right) \text {. }
$$

We have $\mathcal{J}(x) x=\mathcal{A}(x, x) x=0$. We prove Assertion (1) by computing:

$$
0=\mathcal{J}(x, y) \mathcal{J}(x, x) x=\mathcal{J}(x, x) \mathcal{J}(x, y) x=-\frac{1}{2} \mathcal{J}(x) \mathcal{J}(x) y .
$$

Since the Jacobi operator is nilpotent, $\{0\}$ is the only eigenvalue of $\mathcal{J}$. This shows that $A$ is Osserman.

If $p=0$, then $\mathcal{J}(x)$ is diagonalizable. Consequently, $\mathcal{J}(x)^{2}=0$ implies $\mathcal{J}(x)=0$ for all $x$. It now follows $A=0$. If $p=1$, then $A$ is Osserman implies $A$ has 
constant sectional curvature 2, 8. Since $\mathcal{J}(x)^{2}=0$, this again implies $A=0$. This completes the proof of Theorem 1.4

In fact, it is possible to work in a slightly more general setting. Following Bokan [4, one says that $\mathcal{C}$ is a generalized curvature operator if it has the symmetries of the curvature operator defined by a torsion free connection, i.e. if

$$
\begin{aligned}
& \mathcal{C}(x, y) z=-\mathcal{C}(y, x) z \\
& \mathcal{C}(x, y) z+\mathcal{C}(y, z) x+\mathcal{C}(z, x) y=0
\end{aligned}
$$

The proof given above then generalizes immediately to yield:

Corollary 2.1. If $\mathcal{C}$ is a generalized curvature operator on $V$ which is JacobiTsankov, then $\mathcal{J}_{C}$ is Osserman and $\mathcal{J}_{C}(x)^{2}=0$ for all $x \in V$.

Let $\phi$ be a skew-symmetric endomorphism of $V$. Define

$$
\left.A_{\phi}(x, y, z, w):=\langle\phi y, z\rangle\langle\phi x, w\rangle-\langle\phi x, z\rangle\langle\phi y, w\rangle-2\langle\phi x, y\rangle \phi z, w\right\rangle .
$$

The associated Jacobi operator is then given by

$$
\mathcal{J}_{\phi}(x) y=-3\langle y, \phi x\rangle \phi x .
$$

In the following example, we exhibit an algebraic curvature tensor so that $\mathcal{J}(x)^{2}=0$ for all $x \in V$, but which is not Jacobi-Tsankov. Let $\mathbb{R}^{(p, q)}$ denote Euclidean space with a metric of signature $(p, q)$.

\section{Lemma 2.2.}

(1) There exist skew-symmetric endomorphisms $\left\{\phi_{1}, \phi_{2}\right\}$ of $\mathbb{R}^{(4,4)}$ so that

$$
\phi_{1}^{2}=\phi_{2}^{2}=\phi_{1} \phi_{2}+\phi_{2} \phi_{2}=0, \text { and } \phi_{1} \phi_{2} \neq 0 \text {. }
$$

(2) Set $A=-\frac{1}{3}\left\{A_{\phi_{1}}+A_{\phi_{2}}\right\}$. Then $\mathcal{J}_{A}(x)^{2}=0$ for all $x$. Furthermore, $A$ is not Jacobi-Tsankov.

Proof. We apply Lemma 1.4 .5 of [9] to find a collection $\left\{e_{1}, e_{2}, e_{3}, e_{4}\right\}$ of skewsymmetric endomorphisms of $\mathbb{R}^{(4,4)}$ so that:

$$
e_{1}^{2}=e_{2}^{2}=\mathrm{id}, \quad e_{3}^{2}=e_{4}^{2}=-\mathrm{id}, \quad e_{i} e_{j}+e_{j} e_{i}=0 \text { for } i \neq j .
$$

Set $\phi_{1}=e_{1}+e_{3}, \phi_{2}=e_{2}+e_{4}$. These are skew-symmetric endomorphisms with

$$
\phi_{1}^{2}=\phi_{2}^{2}=0, \quad \phi_{1} \phi_{2}+\phi_{2} \phi_{1}=0 \text {. }
$$

Suppose that

$$
\alpha:=\phi_{1} \phi_{2}=\left(e_{1}+e_{3}\right)\left(e_{2}+e_{4}\right)=0 .
$$

We argue for a contradiction. Conjugating by $e_{1}$ yields

$$
e_{1} \alpha e_{1}=\left(-e_{1}+e_{3}\right)\left(e_{2}+e_{4}\right)=0 \text {. }
$$

Adding this equation to the previous one implies $e_{3}\left(e_{2}+e_{4}\right)=0$. Multiplying by $e_{3}$ implies $e_{2}+e_{4}=0$. Conjugating this identity by $e_{2}$ yields $e_{2}-e_{4}=0$ and thus $e_{2}=0$. This is not possible. Assertion (1) now follows.

To prove Assertion (2), we compute:

$$
\begin{aligned}
& \mathcal{J}_{A}(x) y=\left\langle y, \phi_{1} x\right\rangle \phi_{1} x+\left\langle y, \phi_{2} x\right\rangle \phi_{2} x \\
& \mathcal{J}_{A}\left(x_{1}\right) \mathcal{J}_{A}\left(x_{2}\right) y=\left\langle y, \phi_{1} x_{2}\right\rangle\left\langle\phi_{1} x_{2}, \phi_{1} x_{1}\right\rangle \phi_{1} x_{1}+\left\langle y, \phi_{1} x_{2}\right\rangle\left\langle\phi_{1} x_{2}, \phi_{2} x_{1}\right\rangle \phi_{2} x_{1} \\
& \quad+\left\langle y, \phi_{2} x_{2}\right\rangle\left\langle\phi_{2} x_{2}, \phi_{1} x_{1}\right\rangle \phi_{1} x_{1}+\left\langle y, \phi_{2} x_{2}\right\rangle\left\langle\phi_{2} x_{2}, \phi_{2} x_{1}\right\rangle \phi_{2} x_{1} \\
& \quad=\left\langle y, \phi_{1} x_{2}\right\rangle\left\langle\phi_{1} x_{2}, \phi_{2} x_{1}\right\rangle \phi_{2} x_{1}+\left\langle y, \phi_{2} x_{2}\right\rangle\left\langle\phi_{2} x_{2}, \phi_{1} x_{1}\right\rangle \phi_{1} x_{1} .
\end{aligned}
$$

Since

$$
\left\langle\phi_{1} x, \phi_{2} x\right\rangle=-\left\langle\phi_{2} \phi_{1} x, x\right\rangle=\left\langle\phi_{1} \phi_{2} x, x\right\rangle=-\left\langle\phi_{2} x, \phi_{1} x\right\rangle,
$$

we have $\mathcal{J}(x) \mathcal{J}(x)=0$ as desired. 
Choose $x_{1}$ so $\phi_{2} \phi_{1} x_{1} \neq 0$. Set $y=\phi_{1} x_{1}$. We then have:

$$
\begin{aligned}
\mathcal{J}_{A}\left(x_{1}\right) \mathcal{J}_{A}\left(x_{2}\right) y & =\left\langle\phi_{1} x_{1}, \phi_{1} x_{2}\right\rangle\left\langle\phi_{1} x_{2}, \phi_{2} x_{1}\right\rangle \phi_{2} x_{1} \\
& +\left\langle\phi_{1} x_{1}, \phi_{2} x_{2}\right\rangle\left\langle\phi_{2} x_{2}, \phi_{1} x_{1}\right\rangle \phi_{1} x_{1} \\
& =\left\langle\phi_{1} x_{1}, \phi_{2} x_{2}\right\rangle \phi_{1} x_{1}, \\
\mathcal{J}_{A}\left(x_{2}\right) \mathcal{J}_{A}\left(x_{1}\right) y & =\left\langle\phi_{1} x_{1}, \phi_{1} x_{1}\right\rangle\left\langle\phi_{1} x_{1}, \phi_{2} x_{2}\right\rangle \phi_{2} x_{2} \\
& +\left\langle\phi_{1} x_{1}, \phi_{2} x_{1}\right\rangle\left\langle\phi_{2} x_{1}, \phi_{1} x_{2}\right\rangle \phi_{1} x_{2} \\
& =0 .
\end{aligned}
$$

Choose $x_{2}$ so $\left\langle\phi_{1} x_{1}, \phi_{2} x_{2}\right\rangle \neq 0$. Then $\mathcal{J}_{A}\left(x_{1}\right) \mathcal{J}_{A}\left(x_{2}\right) y \neq 0=\mathcal{J}_{A}\left(x_{2}\right) \mathcal{J}_{A}\left(x_{1}\right) y$.

\section{2-STEP JACOBI NILPOTENT ALGEBRAIC CURVATURE TENSORS}

Theorem 1.6 will follow from the following result:

Lemma 3.1. Let $\mathfrak{M}:=(V,\langle\cdot, \cdot\rangle, A)$ be Jacobi-Tsankov. Suppose that there exist $x, y \in V$ so that $\mathcal{J}(x) \mathcal{J}(y) \neq 0$.

(1) There exists $w \in V$ so that

$$
\langle\mathcal{J}(x) \mathcal{J}(y) w, w\rangle=\langle\mathcal{J}(y) \mathcal{J}(w) x, x\rangle=\langle\mathcal{J}(w) \mathcal{J}(x) y, y\rangle \neq 0 .
$$

(2) Let $\mathcal{J}_{x}:=\mathcal{J}(x), \mathcal{J}_{y}:=\mathcal{J}(y)$ and $\mathcal{J}_{x y}:=\mathcal{J}(x, y)$. Set

$$
\begin{array}{llll}
e_{2}=\mathcal{J}_{x} \mathcal{J}_{y} w, & e_{3}=\mathcal{J}_{x} w, & e_{4}=\mathcal{J}_{y} w, & e_{5}=\mathcal{J}_{x y} w \\
f_{2}=\mathcal{J}_{y} \mathcal{J}_{w} x, & f_{3}=\mathcal{J}_{y} x, & f_{4}=\mathcal{J}_{w} x, & f_{5}=\mathcal{J}_{y w} x \\
g_{2}=\mathcal{J}_{w} \mathcal{J}_{x} y, & g_{3}=\mathcal{J}_{w} y, & g_{4}=\mathcal{J}_{x} y, & g_{5}=\mathcal{J}_{w x} y .
\end{array}
$$

The set $S:=\left\{w, x, y, e_{2}, \ldots, e_{5}, f_{2}, \ldots, f_{5}, g_{2}, \ldots, g_{4}\right\}$ is linearly independent.

(3) $e_{5}+f_{5}+g_{5}=0$.

(4) $\operatorname{dim}(V) \geq 14$.

Proof. Choose $w$ so that $e_{2}:=\mathcal{J}(x) \mathcal{J}(y) w \neq 0$. Choose $f$ so $\left\langle e_{2}, f\right\rangle \neq 0$. Set $w(\varepsilon):=w+\varepsilon f$ and $e_{2}(\varepsilon):=\mathcal{J}(x) \mathcal{J}(y) w(\varepsilon)$. Then

$$
p(\varepsilon):=\left\langle w(\varepsilon), e_{2}(\varepsilon)\right\rangle=\left\langle w, e_{2}\right\rangle+2 \varepsilon\left\langle e_{2}, f\right\rangle+\varepsilon^{2}\langle\mathcal{J}(x) \mathcal{J}(y) f, f\rangle .
$$

As $\left\langle e_{2}, f\right\rangle \neq 0, p(\varepsilon)$ is a non-trivial polynomial in $\varepsilon$. Thus it is non-zero for a suitable choice of $\varepsilon$. Thus we may choose $w$ so that $\langle w, \mathcal{J}(x) \mathcal{J}(y) w\rangle \neq 0$. Now,

$$
\begin{aligned}
\langle\mathcal{J}(y) \mathcal{J}(w) x, x\rangle & =-2\langle\mathcal{J}(y) \mathcal{J}(w, x) w, x\rangle=-2\langle\mathcal{J}(y) w, \mathcal{J}(w, x) x\rangle \\
& =\langle\mathcal{J}(y) w, \mathcal{J}(x) w\rangle=\langle\mathcal{J}(x) \mathcal{J}(y) w, w\rangle .
\end{aligned}
$$

Similarly, $\langle\mathcal{J}(w) \mathcal{J}(x) y, y\rangle=\langle\mathcal{J}(x) \mathcal{J}(y) w, w\rangle$ and Assertion (1) follows.

Because $\mathcal{J}(x+\varepsilon y) \mathcal{J}(x+\varepsilon y)=0$ for every $\varepsilon \in \mathbb{R}$ and because $\mathfrak{M}$ is JacobiTsankov, we have the following relations:

$$
\begin{aligned}
& \mathcal{J}_{x}^{2}=0, \quad \mathcal{J}_{y}^{2}=0, \quad \mathcal{J}_{x} \mathcal{J}_{y}=\mathcal{J}_{y} \mathcal{J}_{x}, \\
& \mathcal{J}_{x} \mathcal{J}_{x y}=\mathcal{J}_{x y} \mathcal{J}_{x}=0, \quad \mathcal{J}_{y} \mathcal{J}_{x y}=\mathcal{J}_{x y} \mathcal{J}_{y}=0, \quad \mathcal{J}_{x y}^{2}=-\frac{1}{2} \mathcal{J}_{x} \mathcal{J}_{y} .
\end{aligned}
$$

We have $\mathcal{J}_{w} \mathcal{J}_{y} x \neq 0$ and $\mathcal{J}_{w} \mathcal{J}_{x} y \neq 0$ by Assertion (1). To prove Assertion (2), suppose there is a non-trivial dependence relation among the elements of $S$ :

$$
\begin{aligned}
0 & =a_{1} w+a_{2} e_{2}+a_{3} e_{3}+a_{4} e_{4}+a_{5} e_{5} \\
& +b_{1} x+b_{2} f_{2}+b_{3} f_{3}+b_{4} f_{4}+b_{5} f_{5} \\
& +c_{1} y+c_{2} g_{2}+c_{3} g_{3}+c_{4} g_{4}+c_{5} g_{5} \\
& =a_{1} w+a_{2} \mathcal{J}_{x} \mathcal{J}_{y} w+a_{3} \mathcal{J}_{x} w+a_{4} \mathcal{J}_{y} w+a_{5} \mathcal{J}_{x y} w \\
& +b_{1} x+b_{2} \mathcal{J}_{y} \mathcal{J}_{w} x+b_{3} \mathcal{J}_{y} x+b_{4} \mathcal{J}_{w} x+b_{5} \mathcal{J}_{y w} x \\
& +c_{1} y+c_{2} \mathcal{J}_{w} \mathcal{J}_{x} y+c_{3} \mathcal{J}_{w} y+c_{4} \mathcal{J}_{x} y+c_{5} \mathcal{J}_{w x} y
\end{aligned}
$$

Since we are not taking $g_{5}$, we must set

$$
c_{5}=0 \text {. }
$$


We can apply $J_{x} J_{y}$ to Equation (3.a) to see $a_{1} e_{5}=0$. Since $e_{5} \neq 0, a_{1}=0$. Similarly $b_{1}=c_{1}=0$. If we now apply $J_{x}$ to Equation (3.a), we see

$$
\begin{aligned}
& a_{4} \mathcal{J}_{x} \mathcal{J}_{y} w+c_{3} \mathcal{J}_{x} \mathcal{J}_{w} y=0 \quad \text { so } \\
& 0=\left\langle a_{4} \mathcal{J}_{x} \mathcal{J}_{y} w+c_{3} \mathcal{J}_{x} \mathcal{J}_{w} y, w\right\rangle=a_{4}\left\langle\mathcal{J}_{x} \mathcal{J}_{y} w, w\right\rangle .
\end{aligned}
$$

Since $\left\langle\mathcal{J}_{x} \mathcal{J}_{y} w, w\right\rangle \neq 0, a_{4}=0$. Similarly, we get $a_{3}=b_{3}=b_{4}=c_{3}=c_{4}=0$. Thus Equation (3.a) simplifies to become

$$
0=a_{2} \mathcal{J}_{x} \mathcal{J}_{y} w+a_{5} \mathcal{J}_{x y} w+b_{2} \mathcal{J}_{y} \mathcal{J}_{w} x+b_{5} \mathcal{J}_{y w} x+c_{2} \mathcal{J}_{w} \mathcal{J}_{x} y+c_{5} \mathcal{J}_{w x} y
$$

Applying $\mathcal{J}_{x y}$ then yields

$$
\begin{aligned}
0 & =a_{5} \mathcal{J}_{x y}^{2} w+b_{5} \mathcal{J}_{x y} \mathcal{J}_{y w} x+c_{5} \mathcal{J}_{x y} \mathcal{J}_{w x} y \\
& =\left(a_{5} \mathcal{J}_{x y}^{2}+\frac{1}{4}\left(b_{5}+c_{5}\right) \mathcal{J}_{x} \mathcal{J}_{y}\right) w \\
& =\left(a_{5}-\frac{1}{2}\left(b_{5}+c_{5}\right)\right) \mathcal{J}_{x y}^{2} w
\end{aligned}
$$

This shows $a_{5}=\frac{1}{2}\left(b_{5}+c_{5}\right)$ or $a_{5}=b_{5}=c_{5}$. By Equation (3.b), we have $a_{5}=b_{5}=0$. Taking the inner product with $x, y$, and $w$ then yields, respectively $b_{2}=0, c_{2}=0$, and $a_{2}=0$, which completes the proof of Assertion (2).

To prove Assertion (3), we compute:

$$
\begin{aligned}
& e_{5}+f_{5}+g_{5}=\mathcal{J}_{x y} w+\mathcal{J}_{y w} x+\mathcal{J}_{w x} y \\
= & \frac{1}{2}\{\mathcal{R}(w, x) y+\mathcal{R}(w, y) x+\mathcal{R}(x, y) w+\mathcal{R}(x, w) y+\mathcal{R}(y, w) x+\mathcal{R}(y, x) w\} \\
= & 0 .
\end{aligned}
$$

Assertion (4) is immediate from Assertion (2).

The following example in signature $(8,6)$ was motivated by the proof of Lemma 3.1 It shows the inequality $\operatorname{dim}(V) \leq 13$ in Theorem 1.6 is sharp. The proof is a computer assisted calculation which we omit in the interest of brevity. Details are available upon request from the first author.

Lemma 3.2. Let $\left\{e_{1}, \ldots, e_{4}, \bar{e}_{1}, \ldots, \bar{e}_{4}, \tilde{e}_{1}, \ldots, \tilde{e}_{4}, f_{1}, f_{2}\right\}$ be a basis for a 14 dimensional vector space $V$. Relative to this basis, define an inner product $\langle\cdot, \cdot\rangle$ and an algebraic curvature tensor $A$ on $V$ whose non-zero components are given up to the usual $\mathbb{Z}_{2}$ symmetries by:

$$
\begin{aligned}
& \left\langle e_{1}, e_{2}\right\rangle=\left\langle e_{3}, e_{4}\right\rangle=\left\langle\bar{e}_{1}, \bar{e}_{2}\right\rangle=\left\langle\bar{e}_{3}, \bar{e}_{4}\right\rangle=\left\langle\tilde{e}_{1}, \tilde{e}_{2}\right\rangle=\left\langle\tilde{e}_{3}, \tilde{e}_{4}\right\rangle=1, \\
& \left\langle f_{1}, f_{1}\right\rangle=\left\langle f_{2}, f_{2}\right\rangle=-\frac{1}{2}, \quad\left\langle f_{1}, f_{2}\right\rangle=\frac{1}{4}, \\
& A\left(e_{1}, \tilde{e}_{1}, \tilde{e}_{1}, e_{3}\right)=A\left(e_{1}, \bar{e}_{1}, \bar{e}_{1}, e_{4}\right)=1, A\left(\bar{e}_{1}, e_{1}, e_{1}, \bar{e}_{3}\right)=A\left(\bar{e}_{1}, \tilde{e}_{1}, \tilde{e}_{1}, \bar{e}_{4}\right)=1, \\
& A\left(\tilde{e}_{1}, e_{1}, e_{1}, \tilde{e}_{3}\right)=A\left(\tilde{e}_{1}, \bar{e}_{1}, \bar{e}_{1}, \tilde{e}_{4}\right)=1, \\
& A\left(e_{1}, \bar{e}_{1}, \tilde{e}_{1}, f_{1}\right)=A\left(e_{1}, \tilde{e}_{1}, \bar{e}_{1}, f_{1}\right)=A\left(\bar{e}_{1}, \tilde{e}_{1}, e_{1}, f_{2}\right)=A\left(\bar{e}_{1}, e_{1}, \tilde{e}_{1}, f_{2}\right)=-\frac{1}{2} .
\end{aligned}
$$

Then $\mathfrak{M}:=(V,\langle\cdot, \cdot\rangle, A)$ is Jacobi-Tsankov, $\mathfrak{M}$ has signature $(8,6)$, and $\mathfrak{M}$ is not 2-step Jacobi nilpotent.

\section{The ClAssification of IndeComposable 2-SteP JACOBi NilPotent ALGEBRAIC CURVATURE TENSORS}

In this section, we prove Theorem 1.9 The following Lemma shows that Assertion (3) implies Assertion (2) in Theorem 1.9

Lemma 4.1. Let $\mathfrak{M}$ be as in Definition 1.8. Then $\mathfrak{M}$ is indecomposable and 2-step skew-curvature nilpotent.

Proof. Suppose there is a non-trivial decomposition $\mathfrak{M}=\mathfrak{M}_{1} \oplus \mathfrak{M}_{2}$. This would then induce a non-trivial decomposition of $\left(W, A_{W}\right)$. Since $\left(W, A_{W}\right)$ is assumed indecomposable, either $W \subset V_{1}$ or $W \subset V_{2}$; we suppose without loss of generality that $W \subset V_{1}$. Since $V_{2} \perp V_{1}$ and $W \subset V_{1}, V_{2} \perp W$ so $V_{2} \subset W^{\perp}=W$. Thus $V_{2}$ 
is totally isotropic which is false. This shows $\mathfrak{M}$ is indecomposible. The following argument shows that $\mathfrak{M}$ is 2-step curvature nilpotent. Choose a basis $\left\{e_{i}\right\}$ for $W$ and choose a basis $\left\{\bar{e}_{i}\right\}$ for $\bar{W}$ so the only non-zero components of the inner product are $\left\langle e_{i}, \bar{e}_{j}\right\rangle=\delta_{i j}$. We have

$$
\mathcal{A}\left(e_{i}, e_{j}\right) e_{k}=\sum_{l} A_{W}\left(e_{i}, e_{j}, e_{k}, e_{l}\right) \bar{e}_{l},
$$

while $\mathcal{A}\left(e_{i}, e_{j}\right) e_{k}=0$ if any entry belongs to $\bar{W}$.

We now show Assertion (2) implies Assertion (1) in Theorem 1.9.

Lemma 4.2. Let $\mathfrak{M}:=(V,\langle\cdot, \cdot\rangle, A)$. If $\mathfrak{M}$ is 2-step skew-curvature nilpotent, then $\mathfrak{M}$ is 2-step Jacobi nilpotent.

Proof. Suppose $A$ is a 2-step skew-curvature nilpotent algebraic curvature tensor. Then $\mathcal{A}\left(x_{1}, x_{2}\right) \mathcal{A}\left(x_{3}, x_{4}\right)=0$ for all $x_{1}, x_{2}, x_{3}, x_{4} \in V$. Hence

$$
\begin{gathered}
0=-\left\langle\mathcal{A}\left(x_{1}, x_{2}\right) \mathcal{A}\left(x_{3}, x_{4}\right) x_{4}, x_{2}\right\rangle=-\left\langle\mathcal{A}\left(x_{1}, x_{2}\right) \mathcal{J}\left(x_{4}\right) x_{3}, x_{2}\right\rangle \\
=-\left\langle\mathcal{J}\left(x_{4}\right) x_{3}, \mathcal{A}\left(x_{1}, x_{2}\right) x_{2}\right\rangle=\left\langle\mathcal{J}\left(x_{4}\right) x_{3}, \mathcal{J}\left(x_{2}\right) x_{1}\right\rangle \\
=\left\langle\mathcal{J}\left(x_{2}\right) \mathcal{J}\left(x_{4}\right) x_{3}, x_{1}\right\rangle .
\end{gathered}
$$

Before completing the proof of Theorem 1.9 we must establish a technical result.

Lemma 4.3. Let $\mathfrak{M}:=(V,\langle\cdot, \cdot\rangle, A)$. Suppose that $\mathcal{J}(x) y=0$ for all $x \in V$. Then $A\left(x_{1}, x_{2}, x_{3}, y\right)=0$ for all $x_{i} \in V$.

Proof. We compute:

$$
\begin{aligned}
& A\left(x_{1}, x_{2}, x_{3}, y\right)+A\left(x_{1}, x_{3}, x_{2}, y\right)=2\left\langle\mathcal{J}\left(x_{2}, x_{3}\right) x_{1}, y\right\rangle \\
= & 2\left\langle x_{1}, \mathcal{J}\left(x_{2}, x_{3}\right) y\right\rangle=0 .
\end{aligned}
$$

Consequently $A\left(x_{1}, x_{2}, x_{3}, y\right)=-A\left(x_{1}, x_{3}, x_{2}, y\right)$ for all $x_{i} \in V$. This implies

$$
\begin{aligned}
0 & =A\left(x_{1}, x_{2}, x_{3}, y\right)+A\left(x_{2}, x_{3}, x_{1}, y\right)+A\left(x_{3}, x_{1}, x_{2}, y\right) \\
& =A\left(x_{1}, x_{2}, x_{3}, y\right)-A\left(x_{2}, x_{1}, x_{3}, y\right)-A\left(x_{1}, x_{3}, x_{2}, y\right) \\
& =A\left(x_{1}, x_{2}, x_{3}, y\right)+A\left(x_{1}, x_{2}, x_{3}, y\right)+A\left(x_{1}, x_{2}, x_{3}, y\right) \\
& =3 A\left(x_{1}, x_{2}, x_{3}, y\right) .
\end{aligned}
$$

We complete our discussion by showing that Assertion (1) implies Assertion (3) in Theorem [1.9. Suppose that $\mathfrak{M}$ is indecomposable and that $\mathfrak{M}$ is 2-step Jacobi nilpotent. Set

$$
\bar{W}:=\operatorname{Span}_{v_{1}, v_{2} \in V}\left\{\mathcal{J}\left(v_{1}\right) v_{2}\right\} \quad \text { and } \quad U:=\left\{v \in V: \mathcal{J}\left(v_{1}\right) v=0 \forall v_{1} \in V\right\} .
$$

Then by assumption, $\bar{W} \subset U$. Furthermore, by Lemma 4.3 $A\left(v_{1}, v_{2}, v_{3}, v_{4}\right)=0$ if any of the $v_{i} \in U$. Choose a complementary subspace $W_{1}$ so that $V=U \oplus W_{1}$.

If $\bar{w} \in \bar{W}$, then $\bar{w}=\sum_{j} \mathcal{J}\left(x_{j}\right) y_{j}$. Thus if $u \in U$,

$$
\langle\bar{w}, u\rangle=\left\langle\sum_{j} \mathcal{J}\left(x_{j}\right) y_{j}, u\right\rangle=\sum_{j}\left\langle y_{j}, \mathcal{J}\left(x_{j}\right) u\right\rangle=0 .
$$

Since the metric is non-degenerate, there must exist $\tilde{w} \in W_{1}$ so $\langle\tilde{w}, \bar{w}\rangle \neq 0$. Thus the natural map $W_{1} \rightarrow \bar{W}^{*}$ defined by $\langle\cdot, \cdot\rangle$ is surjective. Let $\left\{\bar{w}_{1}, \ldots, \bar{w}_{k}\right\}$ be a basis for $\bar{W}$. Choose elements $\left\{\tilde{w}_{1}, \ldots, \tilde{w}_{k}\right\}$ in $W_{1}$ so

$$
\left\langle\tilde{w}_{i}, \bar{w}_{j}\right\rangle=\delta_{i j} \text {. }
$$

Suppose that $\left\{\tilde{w}_{1}, \ldots, \tilde{w}_{k}\right\}$ do not span $W_{1}$. We may then choose $0 \neq \tilde{w} \in W_{1}$ so that $\tilde{w} \perp \bar{W}$. Since $\tilde{w} \notin U$, there exists $y$ so that $\mathcal{J}(y) \tilde{w} \neq 0$. Choose $z \in V$ so

$$
0 \neq\langle\mathcal{J}(y) \tilde{w}, z\rangle=\langle\tilde{w}, \mathcal{J}(y) z\rangle .
$$

This contradicts the fact that $\tilde{w} \perp \bar{W}$. Thus $\left\{\tilde{w}_{1}, \ldots, \tilde{w}_{k}\right\}$ is a basis for $W_{1}$. We set

$$
w_{i}:=\tilde{w}_{i}-\frac{1}{2} \sum_{j}\left\langle\tilde{w}_{i}, \tilde{w}_{j}\right\rangle \bar{w}_{j} \text { and } W:=\operatorname{Span}\left\{w_{i}\right\} .
$$


Then the relations of Equation (1.a) are satisfied. Furthermore,

$$
V=U \oplus W \text {. }
$$

Let $\left\{\bar{w}_{1}, \ldots, \bar{w}_{k}, \tilde{u}_{1}, \ldots, \tilde{u}_{l}\right\}$ be a basis for $U$. By Equation (4.a), $\left\langle\bar{w}_{i}, \tilde{u}_{j}\right\rangle=0$. Set

$$
u_{i}:=\tilde{u}_{i}-\sum_{j}\left\langle w_{j}, \tilde{u}_{i}\right\rangle \bar{w}_{j}
$$

We then have $\left\langle u_{i}, w_{i}\right\rangle=\left\langle u_{i}, \bar{w}_{i}\right\rangle=0$. Let $T:=\operatorname{Span}\left\{u_{i}\right\}$. Then:

$$
(V,\langle\cdot, \cdot\rangle, A)=\left(W \oplus \bar{W},\left.\langle\cdot, \cdot\rangle\right|_{W \oplus \bar{W}},\left.A\right|_{W} \oplus 0\right) \oplus\left(T,\left.\langle\cdot, \cdot\rangle\right|_{T}, 0\right) .
$$

Since $(V,\langle\cdot, \cdot\rangle, A)$ is indecomposable, $T=\{0\}$.

\section{ACKNOWLEDGMENTS}

The research of M. Brozos-Vázquez was partially supported by project BFM 2003-02949 (Spain). The research of P. Gilkey was partially supported by the Max Planck Institute for the Mathematical Sciences (Leipzig, Germany). It is a pleasure for both authors to acknowledge helpful conversations with C. Dunn and E. Puffini.

\section{REFERENCES}

[1] N. Blažić, Natural curvature operators of bounded spectrum, In press J. Diff. Geo. and Applications.

[2] N. Blažić, N. Bokan, and P. Gilkey, A Note on Osserman Lorentzian manifolds, Bull. London Math. Soc., 29 (1997), 227-230.

[3] N. Blažić, N. Bokan, P. Gilkey, and Z. Rakić, Pseudo-Riemannian Osserman manifolds, Balkan J. Geom. Appl. 2 (1997), 1-12.

[4] N. Bokan, On the complete decomposition of curvature tensors of Riemannian manifolds with symmetric connection, Rendiconti del Circolo Matematico di Palermo XXIX (1990), 331-380.

[5] M. Brozos-Vázquez and P. Gilkey, Manifolds with commuting Jacobi operators, preprint math.DG/0507554

[6] Q. S. Chi, A curvature characterization of certain locally rank one symmetric spaces, J. Differential Geom., 28 (1988), 187-202.

[7] J. C. Díaz-Ramos, E. García-Río, and R. Vázquez-Lorenzo, New examples of Osserman metrics with nondiagonalizable Jacobi operators, in press J. Diff. Geo. and Applications.

[8] E. García-Río, D. Kupeli, and M. E. Vázquez-Abal, On a problem of Osserman in Lorentzian geometry, Differential Geometry and its Applications 7 (1997), 85-100.

[9] P. Gilkey, Geometric Properties of Natural Operators Defined by the Riemann Curvature Tensor, World Scientific (2002).

[10] P. Gilkey, R. Ivanova, and T. Zhang, Higher order Jordan Osserman, Pseudo-Riemannian manifolds, Class and Quantum Gravity 19 (2002), 4543-4551.

[11] P. Gilkey and S. Nikčević, Complete curvature homogeneous pseudo-Riemannian manifolds, Classical and Quantum Gravity 21 (2004), 3755-3770.

[12] Y. Nikolayevsky, Osserman manifolds of dimension 8, Manuscr. Math. 115 (2004), 31-53.

[13] Y. Nikolayevsky, Osserman Conjecture in dimension $n \neq 8,16$, Mat. Annalen 331 (2005), $505-522$.

[14] R. Osserman, Curvature in the eighties, Amer. Math. Monthly 97 (1990), 731-756.

[15] J. M. Overduin and P. S. Wesson, Kaluza-Klein gravity, Phys. Rep. 283 (1997), 303-378.

[16] V. Sahni and Y. Shtanov, Bouncing braneworlds, Physics Letters B, 557 (2003), 1-6.

[17] Y. Tsankov, A characterization of $n$-dimensional hypersurface in $\mathbb{R}^{n+1}$ with commuting curvature operators, Banach Center Publ. 69 (2005), 205-209.

MBY:Department of Geometry and Topology, Faculty of Mathematics, University of Santiago de Compostela, 15782 Santiago de Compostela, Spain

E-mail address: mbrozos@usc.es

PG: Mathematics Department, University of Oregon, Eugene, OR 97403, USA

E-mail address: gilkey@uoregon.edu 